\title{
Proposal of Automatic Trash System of Pet Litter Sheet
}

\author{
Airi Taniguchi ${ }^{\mathrm{a}}$, Yuhki Kitazono ${ }^{\mathrm{a},}{ }^{*}$ \\ ${ }^{a}$ National Institute of Technology, Kitakyushu College,5-20-1 Shii, Kokuraminami-ku, Kitakyushu-city, Fukuoka 802- \\ 0985, Japan \\ *Corresponding Author: kitazono@kct.ac.jp
}

\begin{abstract}
In late years the people who have a pet increase. As a lot of infectious diseases derived from an animal exist, possibilities infected from animals are also increasing is one of the infection routes is excrement. Thus, we suggest a system to fold a pet litter sheet without touching their excrement. This system consists of seven servomotors. This system folds along the creases of the pet sheet. At first, divide the long side of the pet sheet into three sections. Next fold the left and right sides of the pet sheet. Fold the top and bottom of the pet sheet. Finally, dispose of the pet sheets in the trash. This allows pet owners to dispose of pet sheets without having to touch the excrement. This allows pet owners to dispose of pet sheets without touching the waste, which helps prevent infection.
\end{abstract}

Keywords: zoonosis, pet litter sheet, animal excrement.

\section{Introduction}

In 2020, WHO declared COVID-19 pandemic and the number of infected and dead is increasing every day. Human beings have been fighting against various infectious diseases, including SARS and Ebola, and there are more than 200 types of infectious diseases known by the WHO. There is a high possibility that new animal-derived infections will occur in the future. According to the National Survey of Dog and Cat Breeding by the Pet Food Association of Japan, as of October 2020 , the number of dogs raised was about 8.489 million and the number of cats raised was about 9.64 million, indicating that many animals live together ${ }^{(1)}$. Preventive measures are very important to prevent animal-derived infections from spreading in these situations. There are several causes of infection, but we paid attention to the causes of infection when pathogens in excrement are blown up by the wind and inhaled.
To prevent infection, the Ministry of Health, Labour and Welfare recommends that excretion be disposed of promptly ${ }^{(2)}$. However, it is too burdensome to dispose of them every time their wastes are excreted. There, a product called brilliant pads that can automatically dispose of excrement is on sale ${ }^{(3)}$. As the product winds the roll with animal wastes every fixed time when excreted on a roll-like sheet, it is a very innovative product. However, it still have problems. Excrement remains unwound until the roll finish using, and may be spilled if the excrement is not defecated in a certain area of the sheet.

Therefore, we developed a system that automatically folds up pet sheets and throws them away only pressing the button. The system folds pet sheets one by one and throws them away in the trash can, so once excreted, they can be disposed of in the trash without the remains. Furthermore, since it is folded as people do, and disposed of, it can be disposed of wherever excreted

\section{Automatic Trash System of Pet Litter Sheet}

Figure 1 shows configuration of the device of this study. Figure 1(a) is a front view and Fig. 1(b) is a side view. In addition, movable part 1 to movable part 7 in Fig. 1 are parts operated by servo motors.

A user puts the pet sheet on the system as shown in Fig. 2(a), and when a start button is pressed, the pet sheet is automatically folded and thrown into the trash can. A pet sheet is folded according to the procedure shown in Fig. 2. The system fold pet sheet by folding movable part 1 about 140 degrees as shown in Fig. 2(b). After returning movable part 1, fold movable part 1 down to 90 degrees again to prevent the pet sheet from slipping. Fold the pet sheet over about 140 degrees as shown in Fig. 2(c). After restoring Movable part 2 to its original position, lower movable part 2 to 90 degrees again. To make it easier to fold, tilt Movable part 320 degrees 


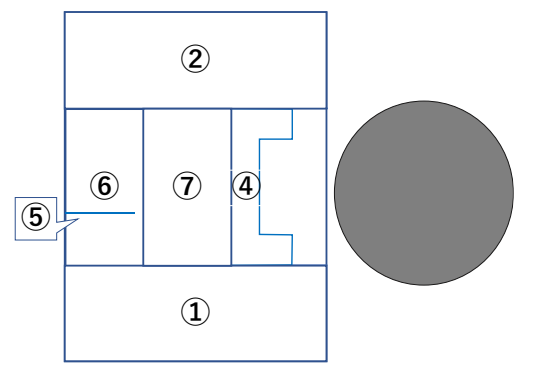

(a) Top view.

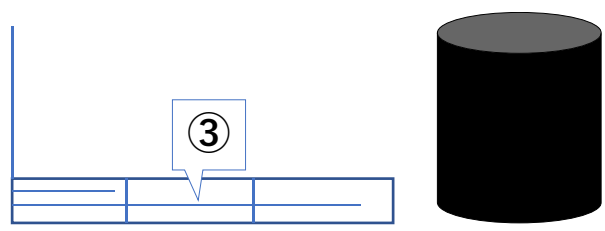

(b) Side view.

Fig. 1. Constitution of system.

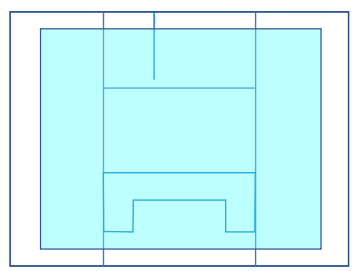

(a) Step 1 .

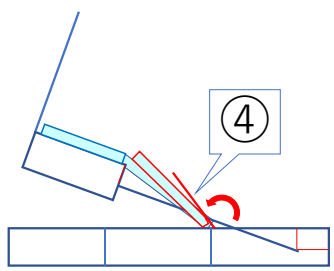

(e) Step 5 .

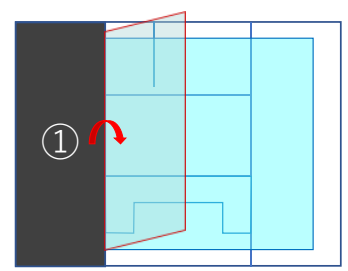

(b) Step 2 .

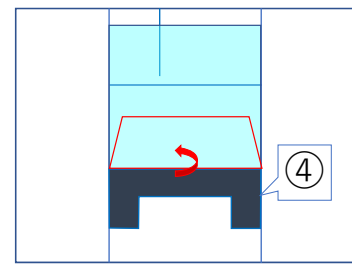

(f) Step 6 .

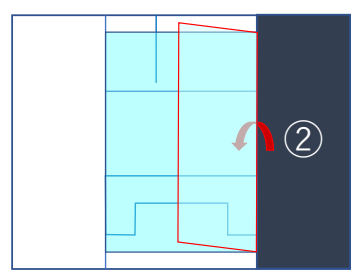

(c) Step 3 .

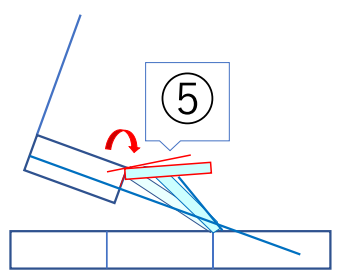

(g) Step 7.

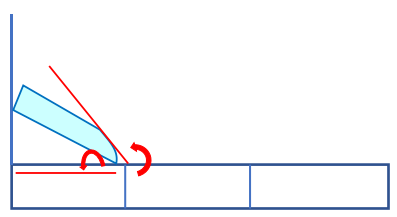

(k) Step 11.

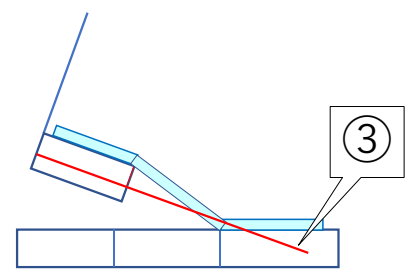

(d) Step 4 .

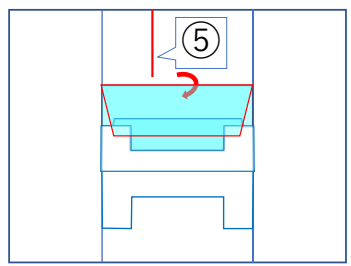

(h) Step 8 .

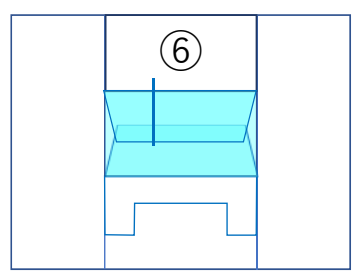

(i) Step 9.

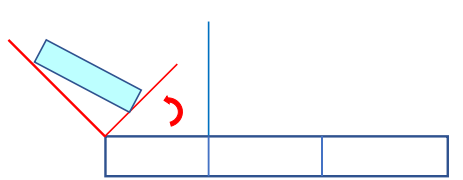

(m) Step 13 .

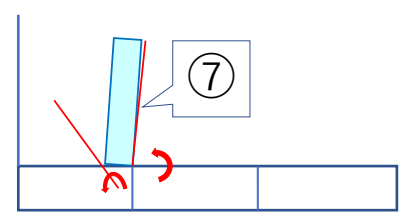

(j) Step 10 .

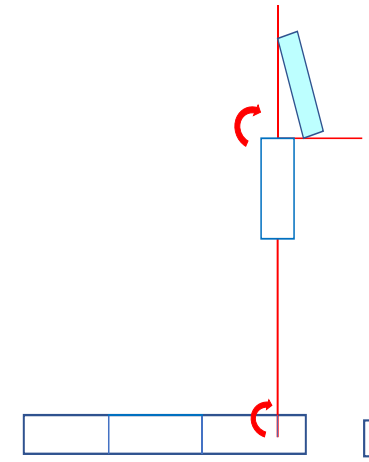

(n) Step 14.

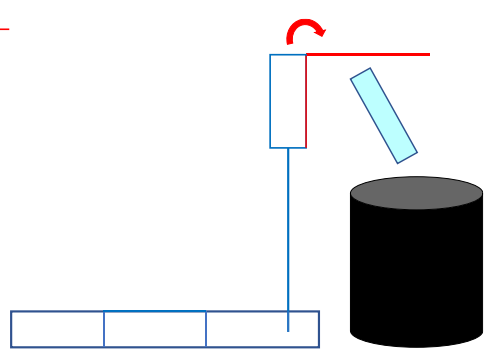

(o) Step 15.

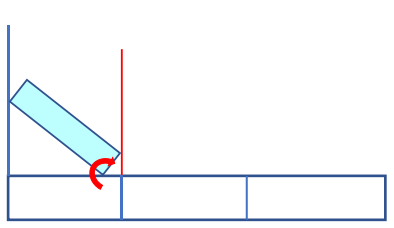

(1) Step 12.

Fig. 2. System movement.

as shown in Fig. 2(d). Folding of the center is performed as follows. Fold movable part 4 about 145 degrees as shown in Fig. 2(e) and fold the pet sheet. A front view at that time is as shown in Fig. 2(f). Since the pet sheet absorbs and inflates urine, fold movable part 5150 degrees instead of returning movable part 4 as shown in Fig. 2(g). A front view at that time is shown in Fig. 2(h). After holding the pet sheet in 5, put movable part 3 and movable part 4 back together. In order to 


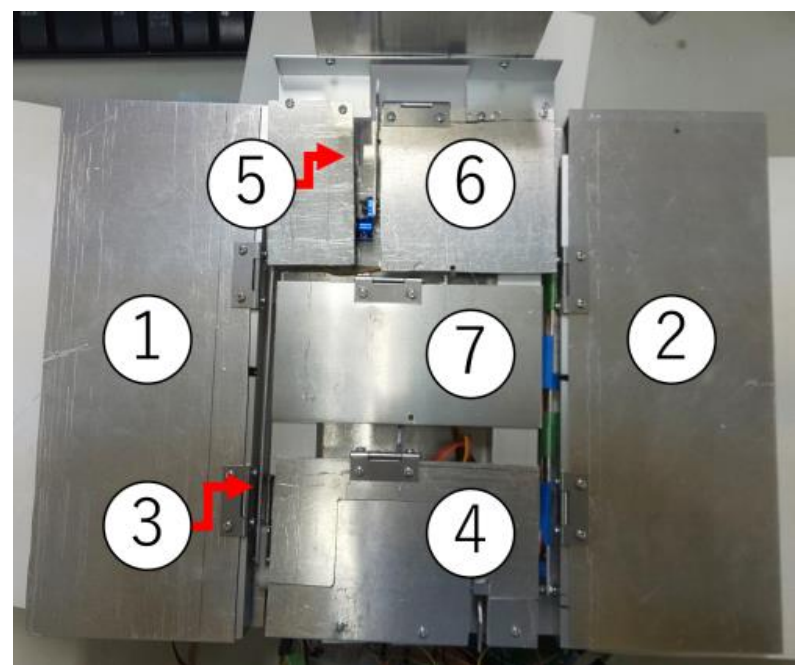

Fig. 3. Prototype unit.

throw away the pet sheets, it is necessary to move the pet sheets to movable part 6 of Fig. 2(i). In order to prevent the pet sheet from being shifted, stop it once at an angle where the pet sheet becomes vertical by pinching it between movable part 5 and movable part 7 as shown in Fig. 2(j). Then, move the pet sheet to movable part 6 as shown in Fig. 2(k). Tilt Movable part 6 outward as shown in Fig. 2(m) to prevent the pet sheet from falling. At that time, movable part 7 is returned to a vertical position so that the pet sheet does not repel as shown in Fig. 2(1). After tilting movable part 6, return movable part 7 to its initial position. Move on to throwing away pet sheets. As pet sheets need to be brought higher than the trash can, lift movable part 6 to a vertical position in movable part 3 as shown in Fig. 2(n). At that time, raise the angle of movable part 6 at the same time to prevent the pet sheet from falling. When lifted up to the vertical position, return the angle movable part 7 to the initial position as shown in Fig. 2(o) and throw the pet sheet away in the trash can. Finally, return movable part 3 to the initial position as shown in Fig. 2(p) and finish the operation.

\section{Prototype}

This section describes the prototype that realizes the system described in Chapter 2. Fig. 3 shows an overall picture of the manufactured system. The size of this system is approximately $400 \mathrm{~W} \times 300 \mathrm{D} \times 70 \mathrm{H}(\mathrm{mm})$. This system consists of servomotors in movable part 1 to 7 . Movable part 3 uses S777FCG/6BB from GWS. For others, S03T2BBMG from GWS was used. Details of each are shown below. A servo motor is arranged as shown in Fig. 4(a). A range of movement of the servo motor is set to 0-140 degrees so that the aluminum plate moves toward the center. As shown in Fig.

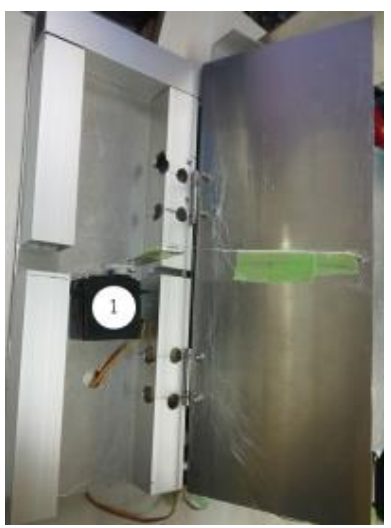

(a) Servo 1

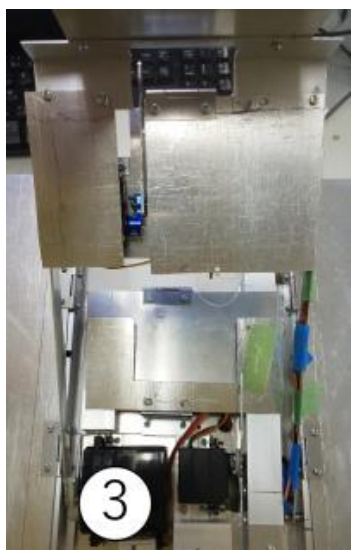

(c) Servo 3.

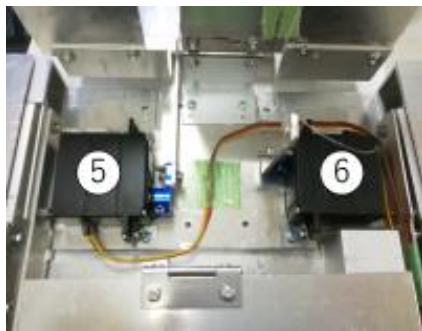

(e) Servo 5, 6 .

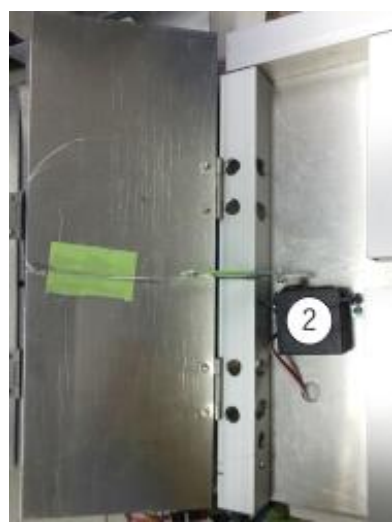

(b) Servo 2 .

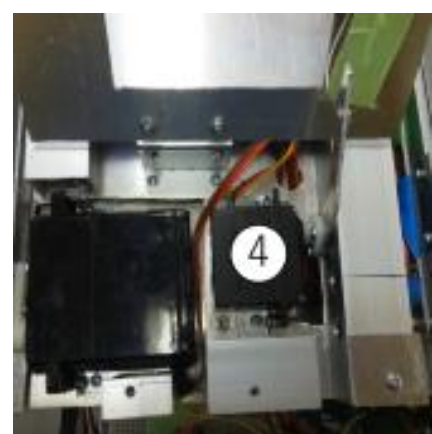

(d) Servo 4

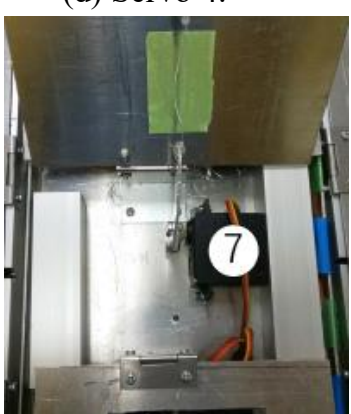

(f) Servo 7.
Fig. 4. Servo layout.

4(b), movable part 1 is inverted left and right, and movable part 3 is arranged with servo motors as shown in Fig. 4(c). An arm of about $300 \mathrm{~mm}$ is attached and a servomotor is installed at the tip in order to assume the function of putting the pet sheet in the trash can. A moving range of the servo motor is set to 0-90 degrees. Because a load is applied to the servo motor, a servo motor having a large torque is used. Fig. 4. Shows the way Movable part 4 is positioned in the servo motor. A range of movement of the servo motor is set from 0 degrees to 145 degrees so that the aluminum plate moves toward movable part 7 . In movable part 5 and movable part 6 , servo motors were installed as shown in Fig. 4(e). In movable part 5 , the range of movement of the servomotor is set from 0 


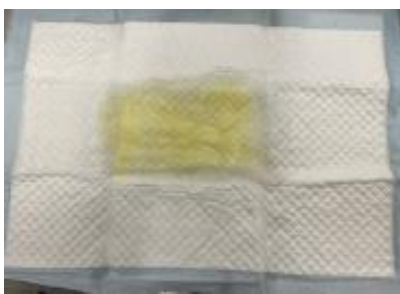

(a) Setting.

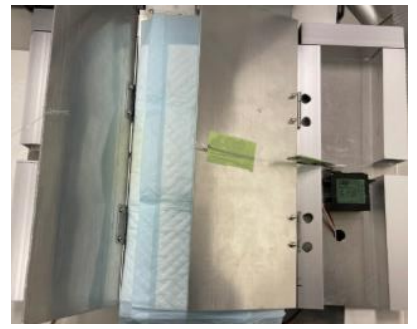

(c) Servo 2 move.

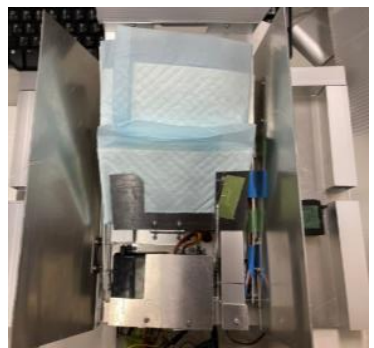

(e) Servo 4 move.

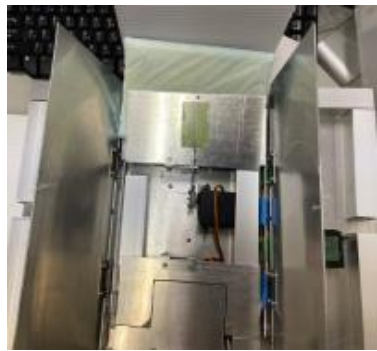

(g) Servo 5, 7 move.

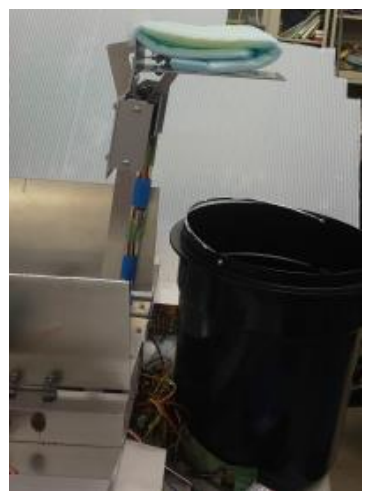

(i) Servo 3, 6 move.

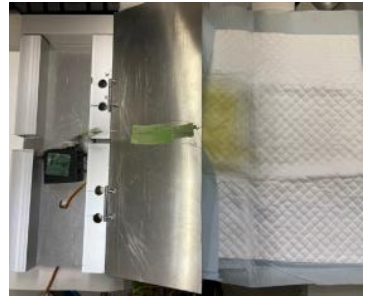

(b) Servo 1 move.

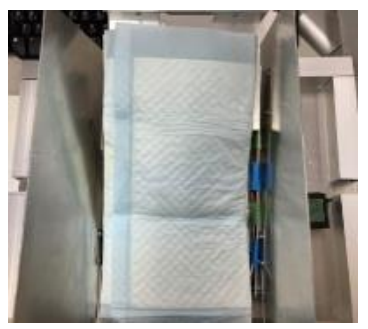

(d) Servo 3 move.

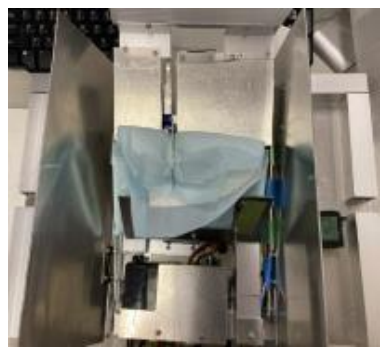

(f) Servo 5 move

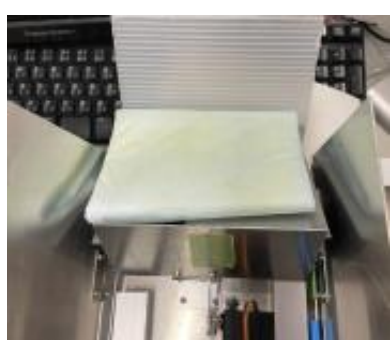

(h) Servo 6 move.

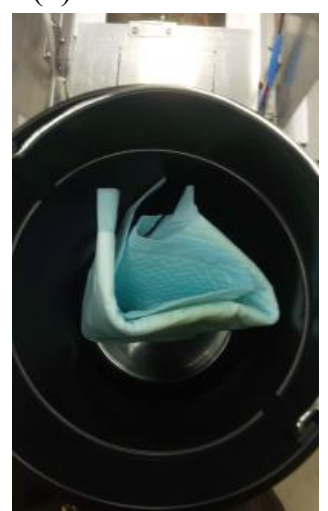

(j) completed.
Fig. 5. Movement was completed.

degrees to 150 degrees so that the arm moves toward movable part 7. In Fig. 2(1), the range of movement of the servo motor is set from 0 degrees to 155 degrees. A servo motor is arranged as shown in Fig. 4(f). A range of movement of the servo motor is set from 0 degrees to 150 degrees so that the aluminum plate moves toward movable part 6 .

Operation of this prototype machine is shown in Fig. 5.

First, a pet sheet is placed as shown in Fig. 5(a). The pet sheets were wet by yellow water in place of urine. At the start of the operation, the left side of the pet sheet is folded as shown in Fig. 5(b) and the right side as shown in Fig. 5(c).

A pet sheet is inclined as shown in Fig. 5(d), the front side of the pet sheet is folded as shown in Fig. 5(e), and the back side is collapsed as shown in Fig. 5(f) and folded. A pet sheet is moved to the back as shown in Fig. $5(\mathrm{~g})$, and the pet sheet is held as shown in Fig. 5(h). Lift the pet sheet to the vertical position as shown in Fig. 5(i). Then throw the pet sheets away in the trash can. The pet sheet in the trash can is shown in Fig. $5(j)$.

\section{Experiment}

Practicality of this system was confirmed through experiments. Because excrement contains urine and feces, the following two experiments were conducted.

Further, success or failure is judged by whether or not the pet sheet is put in the trash can properly.

\subsection{Urine}

Healthy dogs urinate $24-41 \mathrm{ml}$ per kilogram per day, and adult dogs urinate 3-4 times per day ${ }^{(4)(5)}$. The prototype was designed for small dogs, so if the weight of the small dog is calculated as $5 \mathrm{~kg}$, the amount of urination at a time is approximately $40 \mathrm{ml}$. Therefore, $40 \mathrm{ml}$ of colored water was used in one experiment in this time. As shown in Fig. 6, the pet sheet is divided into nine equal parts, colored water in place of urine is applied to each position, and the sheet is folded five times at each position by the above device. The success rates for each location are shown in Table 1.

\subsection{Wastes}

Currently, a product called a pet scoop is on sale, and the width of the product is $9 \mathrm{~cm}$, so the size of the feces is 9 $\mathrm{cm}^{(6)}$. As shown in Fig. 7, the pet sheet is divided into 25 equal parts, clay resembling feces is placed at each position, placed in four directions like Fig. 8 and folded by the above device and discarded. The success rates for each location are shown in Table 2.

Table 1 shows that the success rate of disposing of pet sheets containing urine is $100 \%$, which is sufficient for practical use. Table 2 shows that the success rate for pet 


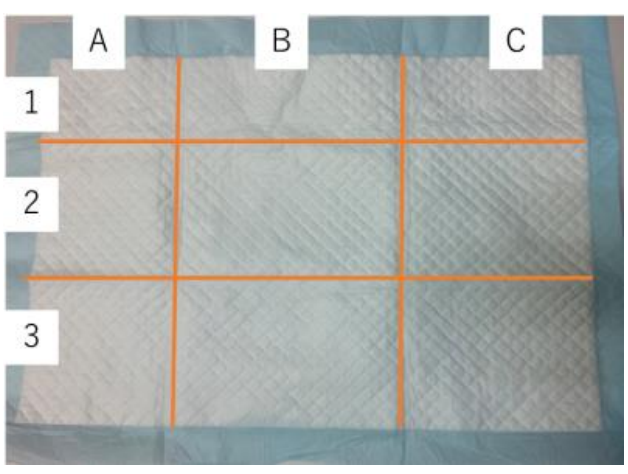

Fig. 6. Position of Urine.

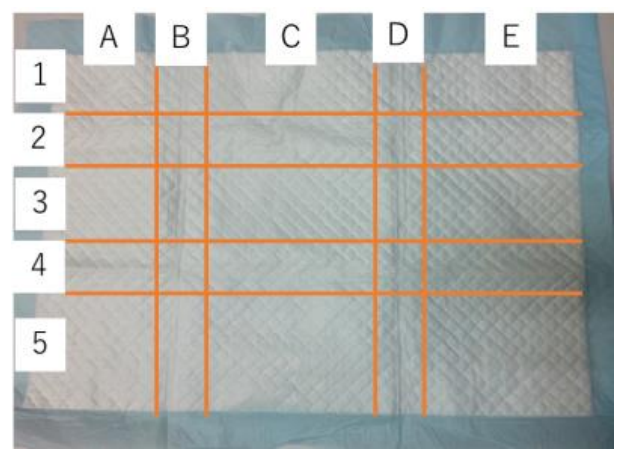

Fig. 7. Position of feces.

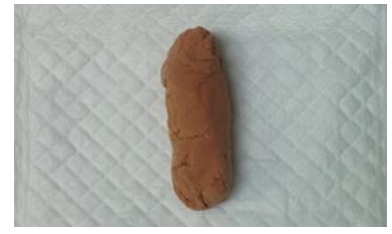

(a) Pattern 1.

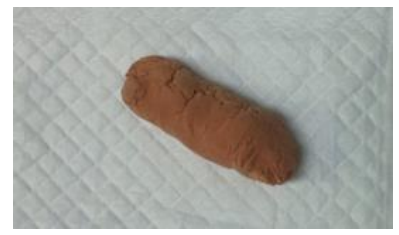

(c) Pattern 3

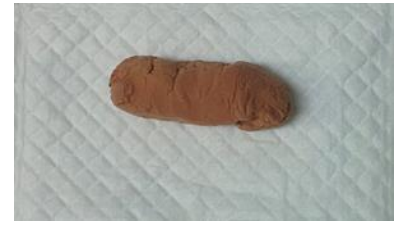

(b) Pattern 2.

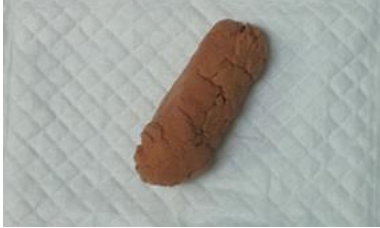

(d) Pattern 4.
Fig. 8. Direction of feces.

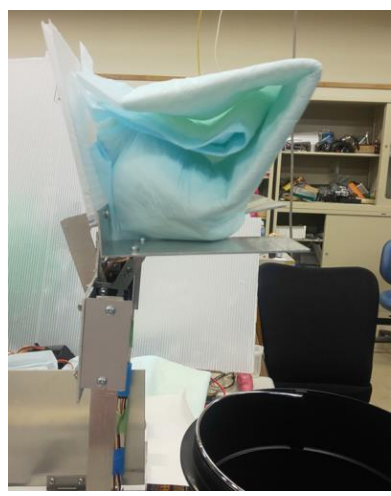

Fig. 9. Defective pattern
Table 1. Success rate for urine [\%].

\begin{tabular}{|c||c|c|c|}
\hline & A & B & C \\
\hline \hline 1 & 100 & 100 & 100 \\
\hline 2 & 100 & 100 & 100 \\
\hline 3 & 100 & 100 & 100 \\
\hline
\end{tabular}

Table 2. Success rate for feces [\%].

\begin{tabular}{|c||c|c|c|c|c|}
\hline & A & B & C & D & E \\
\hline \hline 1 & 100 & 100 & 100 & 100 & 100 \\
\hline 2 & 75 & 100 & 100 & 75 & 100 \\
\hline 3 & 75 & 100 & 100 & 100 & 100 \\
\hline 4 & 100 & 100 & 100 & 100 & 100 \\
\hline 5 & 75 & 100 & 100 & 100 & 100 \\
\hline
\end{tabular}

sheets containing feces is $95 \%$, which is considered to be practical.

As shown in Fig. 9, it could not be folded neatly depending on the position of wastes.

Also, it takes 45 seconds for one operation, so it can be processed quickly.

Reasons for failure in folding pet sheets containing wastes include the pet sheet getting caught in the cut of the arm and the feces falling out during the folding process.

\section{Conclusion}

In this study, an automatic folding system for pet sheets was developed. In the developed robot, the operation from folding the pet sheet to disposal is automatically performed by pressing a switch. Consequently, since a user can discard the pet sheet without touching the pet sheet, the risk of infection to an infectious disease originating from an animal can be greatly reduced.

From now on, we aim to add functions such as automatically detecting and folding excrement using a camera, automatically installing pet sheets, taking pictures of excrement with a camera installed to check the condition of excrement, and attaching them to emails.

\section{References}

(1) Pet Food Association of Japan : "2020 National Survey of Dog and Cat Ownership p.18" https://petfood.or.jp/data/chart2020/3.pdf

(2) Ministry of Health, Labour and Welfare : "Do you know about zoonotic diseases?" https://www.mhlw.go.jp/stf/seisakunitsuite/bunya/00001 55663.html 
(3) OFT Corporation : "Roll-type Automatic Dog Toilet Brilliant Pad" http://oftww.com/item/brilliant

(4) The dog's feelings: "Can you tell me how much urine a dog urinates per day and how long he can hold it?" https://dog.benesse.ne.jp/qa/content/?id=32273

(5) The dog's feelings : "How many times a day is appropriate for a dog to pee? Q \& A about how many times a dog should pee" https://dog.benesse.ne.jp/withdog/content/?id=61329

(6) Azumi Shoji : "Pet Shovel Clean Shovel AZ0874" https://azumi.biz/cargo/shop.cgi?class=14\&keyword=\& $\mathrm{FF}=0$ \&price_sort $=\&$ order 